\title{
Occurrence of Rickettsia felis in dog and cat fleas (Ctenocephalides felis) from Italy
} Gioia Capelli ${ }^{1 *}$, Fabrizio Montarsi ${ }^{1}$, Elena Porcellato ${ }^{1}$, Giulia Maioli ${ }^{2}$,
Carmelo Furnari $^{3}$, Laura Rinaldi ${ }^{4}$, Gaetano Oliva ${ }^{5}$, Domenico Otranto $^{6}$

Address: ${ }^{1}$ Istituto Zooprofilattico Sperimentale delle Venezie, Legnaro, Padova, Italy, ${ }^{2}$ Istituto Zooprofilattico della Lombardia e dell'Emilia Romagna, Reggio Emilia, Italy, ${ }^{3}$ ULSS 20, Verona, Italy, ${ }^{4}$ Department of Pathology and Animal Health, Faculty of Veterinary Medicine, University of Naples "Federico II", Italy, ${ }^{5}$ Department of Veterinary Clinical Science, Faculty of Veterinary Medicine, University of Naples "Federico II", Italy, ${ }^{6}$ Department of Veterinary Public Health and Veterinary Sciences, Faculty of Veterinary Medicine, University of Bari, Italy

Email: Gioia Capelli* - gcapelli@izsvenezie.it; Fabrizio Montarsi - fmontarsi@izsvenezie.it; Elena Porcellato - parassitologia@izsvenezie.it; Giulia Maioli - giulia.maioli@gmail.com; Carmelo Furnari- cfurnari@tiscali.it; Laura Rinaldi - laura.rinaldi@unina.it; Gaetano Olivagaeoliva@unina.it; Domenico Otranto - d.otranto@veterinaria.uniba.it

* Corresponding author

from 4th International Canine Vector-Borne Disease Symposium Seville, Spain. 26-28 March 2009

Published: 20 April 2009

Parasites \& Vectors 2009, 2(SuppI I):S8 doi:I0.I I86/I756-3305-2-SI-S8

This article is available from: http://www.parasitesandvectors.com/content/2/SI/S8

(c) 2009 Capelli et al; licensee BioMed Central Ltd.

This is an Open Access article distributed under the terms of the Creative Commons Attribution License (http://creativecommons.org/licenses/by/2.0), which permits unrestricted use, distribution, and reproduction in any medium, provided the original work is properly cited.

\begin{abstract}
Rickettsia felis is an obligate intracellular bacterium belonging to the spotted fever group, suspected to cause a murine typhus-like illness in humans, with a cosmopolitan distribution. This study was designed to estimate presence and occurrence of this pathogen in fleas collected from dogs and cats in different areas of Italy. Two species of fleas were identified, Ctenocephalides felis (80.3\%) and Ctenocephalides canis (19.7\%).

Overall, 320 fleas (257 C. felis and 63 C. canis) collected from II7 animals (73 dogs and 44 cats) were tested. Thirty-eight (II.9\%) C. felis fleas, I3 from cats (17.6\%) and 25 from dogs (I0.2\%) were positive for R. felis. No C. canis was positive. Fleas from cats showed a tendency to be more positive than fleas from dogs. Prevalence of $R$. felis among areas and within provinces of the same area was extremely variable, ranging from 0 to $35.3 \%$. Overall, prevalence in north-eastern Italy $(23.2 \%)$ was significantly higher than in south-western Italy (7.I\%). This study confirmed the occurrence of $R$. felis in cat and dog fleas (C. felis) from Italy, similar to other European countries. The results also suggest that $R$. felis should be considered in the human differential diagnosis of any spotted-like fever in Italy, especially if the patient is known to have been exposed to flea bites.
\end{abstract}

\section{Findings}

Rickettsia felis is an obligate intracellular bacterium belonging to the spotted fever group, suspected to cause a murine typhus-like illness in humans [1-3]. Following its first detection [4] in midgut cells of the cat flea
Ctenocephalides felis, this organism has been later described as a new species [5].

Biological and genomic investigations have shown that R. felis displays unique features compared with other 
rickettsieae, in particular in its genomic $[6,7]$ and laboratory cultivation [8]. Molecular epidemiological investigations allowed detection of $R$. felis in fleas from different geographical areas of the world, further supporting the cosmopolitan distribution of this pathogen [1].

In Italy, R. felis has been detected in cat and dog fleas limitedly to an area of north-eastern Italy [9]. The aim of the present study was to obtain further information on the occurrence and distribution of $R$. felis in fleas from different Italian regions, where the presence of this agent is still unknown.

From March 2008 to March 2009, fleas were collected from owned animals, colony cats, stray and kennelled dogs of four provinces of Veneto region (north-eastern Italy), two provinces of Campania region (south-western Italy) and of Bari province (Apulia region, south-eastern Italy). All fleas, preserved in isopropanol, were morphologically identified and sexed by using light microscopy and following an identification key [10]. A range of 1-3 fleas per animal host were randomly chosen for molecular analyses.

DNA extraction was performed using a commercial kit (DNeasy ${ }^{\varpi}$ Blood\&Tissue Qiagen kit). A 401-bp fragment of the rickettsial gltA (citrate synthase) gene was PCRamplified using CS-78 (forward) and CS-323 (reverse) primers [11].

Briefly, PCRs $(50 \mu \mathrm{l})$ were performed in an Applied Biosystems Thermocycler (Gene Amp PCR System 9700), adding $5 \mu \mathrm{l}$ of the DNA template to $31.7 \mu \mathrm{l}$ of the molecular-grade water, $5 \mu \mathrm{l}$ of buffer $10 \mathrm{x}, 3 \mu \mathrm{l}$ of $\mathrm{MgCl}_{2} 25$ $\mathrm{mM}, 1 \mu \mathrm{l}$ of dNTPs $10 \mathrm{mM}, 2 \mu \mathrm{l}$ of each primer (CS-78 and CS-323) $10 \mu \mathrm{M}$ and $3 \mu \mathrm{l}$ of AmpliTaq Gold 5U. PCR cycling conditions were as follows: 1 initial cycle at $94^{\circ} \mathrm{C}$ for $10^{\prime}, 35$ cycles of $15^{\prime \prime}$ at $95^{\circ} \mathrm{C}, 30^{\prime \prime}$ at $55^{\circ} \mathrm{C}$, and $30^{\prime \prime}$ at $72^{\circ} \mathrm{C}$, and 1 final step at $72^{\circ} \mathrm{C}$ for 7 .

The complete coding sequences were generated using the Big Dye Terminator v3.1 cycle sequencing kit (Applied Biosystem, Foster City, CA, USA). The products of the sequencing reactions were purified using PERFORMA DTR Ultra 96-Well kit (Edge BioSystems, Gaithersburg, MD, USA) and sequenced in a 16-capillary ABI PRISM $3130 \times 1$ Genetic Analyzer (Applied Biosystem, Foster City, CA, USA). Sequences obtained were compared with those of characterised rickettsieae in Genbank by using BLAST analysis (www.ncbi.nlm.nih.gov).

Prevalence differences in relation to host species (cats and dogs), provenance and flea sex were tested by $\chi^{2}$ test or Fisher's Exact test using the SPSS statistical package (SPSS Inc., USA) for Windows, version 15.0.
Table I - Number of fleas tested and positive for Rickettsia felis and number of animals on which fleas were collected from different geographical areas of Italy.

\begin{tabular}{llcll}
\hline Species & $\begin{array}{l}\text { Province } \\
\text { (area of Italy) }\end{array}$ & $\begin{array}{l}\text { Examined } \\
\text { fleas/animals }{ }^{\text {a }}\end{array}$ & $\begin{array}{l}\text { Positive fleas/ } \\
\text { animals }\end{array}$ & $\begin{array}{l}\text { R. felis } \\
\text { prevalence } \\
\text { in fleas, \% }\end{array}$ \\
\hline Cat & Belluno (ne) & $1 / 1$ & 0 & - \\
& Padova (ne) & $9 / 9$ & $2 / 2$ & 22.2 \\
& Venezia (ne) & $39 / 13$ & $5 / 3$ & 12.8 \\
& Verona (ne) & $17 / 15$ & $6 / 6$ & 35.3 \\
& Napoli (sw) & $8 / 6$ & 0 & 0.0 \\
& Total fleas/cats & $74 / 44$ & $13 / 11$ & 17.6 \\
& & & & \\
Dog & Belluno (ne) & $1 / 1$ & $1 / 1$ & - \\
& Padova (ne) & $34 / 9$ & $10 / 2$ & 29.4 \\
& Venezia (ne) & $6 / 3$ & $1 / 1$ & 16.7 \\
& Verona (ne) & $5 / 2$ & $1 / 1$ & 20.0 \\
& Napoli (sw) & 97 b/29 & $7 / 5$ & 7.2 \\
& Caserta (sw) & 62 c/8 & $5 / 4$ & 8.0 \\
Avellino (sw) & $2 / 1$ & 0 & - \\
Bari (se) & $39 / 20$ & 0 & 0.0 \\
Total fleas/dogs & $246 / 73$ & $25 / 14$ & 10.2 \\
& & & \\
& Total & $320 / 117$ & $38 / 25$ & 11.9 \\
\hline
\end{tabular}

a fleas/animals $=$ number of fleas examined and collected from a number of animals.

b Including 62 Ctenocephalides canis fleas.

c Including one Ctenocephalides canis flea.

se, south-eastern Italy; ne, north-eastern Italy; sw, south-western Italy.

Two species of fleas were identified, C. felis (80.3\%) and Ctenocephalides canis (19.7\%). Cats were infested only by C. felis and dogs by both species, with $C$. felis again as the predominant flea $(75 \%)$.

The results of the molecular study are summarised in Table 1. Overall, 320 fleas (257 C. felis and 63 C. canis) collected from 117 animals (73 dogs and 44 cats) were tested. Thirty-eight (11.9\%) C. felis fleas, 13 from cats $(17.6 \%)$ and 25 from dogs (10.2\%) were positive for $R$. felis yielding PCR bands of the expected size (Figure 1). No C. canis was positive. Sequenced amplicons displayed an overall $99 \%$ similarity to $R$. felis sequence available in the Genbank database (Rickettsia felis URRWXCal2; accession number CP000053.1).

Specifically, positive fleas were found from both dogs and cats in all four provinces of north-eastern Italy, from dogs in two provinces of south-western Italy (Naples and Caserta), whereas all fleas from Bari province (southeastern) were negative (Table 1).

Fleas from cats showed a tendency (Fisher's exact test; $\mathrm{p}=$ 0.068 ) to be more positive (17.6\%) than fleas from dogs $(10.2 \%)$. Male and female fleas showed a similar rate of infection (11.6\% and $8.9 \%$, respectively). Prevalence of $R$. felis among areas and within provinces of the same area 


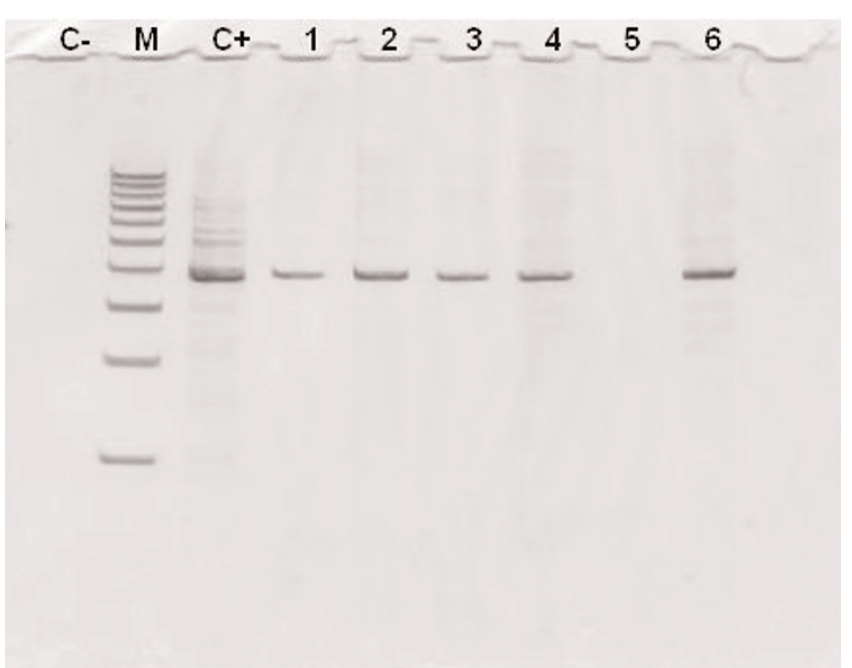

Figure I - Image of an acrylamide gel 7\% showing PCR amplification of the 40 I-bp fragment (gltA rickettsial gene).

$\mathrm{C}+=$ positive control; $\mathrm{C}-=$ negative control (distilled water); $\mathrm{I}-6$ = flea samples ( $\mathrm{I}, 2,3,4,6$ positives); $M=$ molecular weight (I00 bp PCR Molecular Ruler, BioRad, Laboratories Inc., Hercules, CA, USA).

was extremely variable, ranging from 0 to $35.3 \%$ (Table 1). In general and excluding fleas from Bari province (a single site of sampling) prevalence in north-eastern Italy $(23.2 \%)$ was significantly higher than in south-western Italy $(7.1 \%)\left(\chi^{2}\right.$ test $\left.=14.956 ; \mathrm{p}<0.01\right)$.

Our results indicate that $R$. felis is present in C. felis fleas from several geographical locations of Italy. The occurrence of $R$. felis-positive fleas from dogs and cats in north-eastern and south-western Italy is similar to data available in other European countries, i.e. Germany [12], France [13] and Spain [14].

In this study, R. felis was detected for the first time in south-western Italy, whilst the negative results from south-eastern Italy require further investigations, being fleas collected only in dogs housed in a sole municipal kennel. The higher prevalence of $R$. felis-infected fleas in north-eastern Italy compared with south-western areas is currently difficult to explain, a sampling bias can not be excluded and only further and more balanced sampling will confirm this trend.

In our study, C. felis was the only flea species infected as reported by several other authors, nevertheless C. canis was previously found as a $R$. felis carrier in other investigations, along with others flea species, ticks and mites (reviewed in [2]). A possible explanation for the negativity of C. canis in our study could be that this flea was found only in dogs not showing mixed infection with C. felis and presumably not infected with $R$. felis. This hypothesis would explain also why $C$. felis fleas were found more infected when collected on cats rather than on dogs. However, the mechanism of $R$. felis transmission to vertebrates and to uninfected fleas in nature is still unknown, even though there is experimental evidence indicating that $R$. felis is maintained in cat fleas primarily by transovarian and transstadial transmission [15].

Besides vertical transmission, the first identification of the bacterium in salivary glands of $C$. felis [16], along with the evidence of seroconvertion and $R$. felis DNA detection in blood from cats exposed to infected fleas [17], strongly supports the potential for horizontal transmission among vectors and to vertebrate hosts. Among the other possible mammal hosts of C. felis, several studies [3] have shown that opossums seroconvert, and are usually heavily infested with the infected cat fleas. Thus, opossums could indirectly serve as a bridge for the transmission of $R$. felis to vertebrates.

Furthermore, a study conducted in Germany [12] in fleas collected from cats and dogs, provided evidence that Archeopsylla erinacei (the hedgehog flea) carried $R$. felis in all the specimens tested, compared to a low positivity found in C. felis (9\%). The data above suggest that $A$. erinacei could have played a role in transmitting $R$. felis to humans in Germany and indicate the hedgehog as another candidate to be a potential reservoir of the infection.

Opossums and other vertebrate hosts may play a role in rickettsial horizontal transmission to other ectoparasites, and this may account for the occasional reports of $R$. felis infection in other flea species as well as ticks.

Clinical symptoms have not been reported in any animal carrying positive fleas. The current knowledge suggests that the only role of mammals infected by $R$. felis is likely to amplify the cycle by fleas feeding on their $R$. felisinfected blood. This hypothesis is supported by the fact that even if vertical transmission of $R$. felis persists in $C$. felis for at least 12 generations not feeding in a R. felisinfected host, over successive generations prevalence shows a natural decrease [18].

Actually, R. felis has been associated with diseased hosts only in humans. Infection by $R$. felis in humans has been reported in USA, Mexico, Brazil, France and Spain [1,2] both by serological and molecular evidence, but unfortunately the pathogen has never been successfully isolated from humans. The lack of a human isolate of $R$. felis does not permit the definition of this organism as a confirmed human pathogen. 
To our knowledge no human cases of rickettsiosis due to $R$. felis have been diagnosed or suspected in Italy. However, clinical symptoms for $R$. felis infections are similar to those of other rickettsial diseases, which make difficult an aetiological diagnosis based only on the clinical presentations. Conversely, spotted fever rickettsiosis due to $R$. conorii in Italy is endemic in southern regions while some sporadic case of murine typhus due to $R$. typhi has been reported and a few cases were serologically attributed to $R$. helvetica [19]. Recently, $R$. slovaca has been identified in ticks removed from humans showing a tick-borne lymphadenopathy in the Tuscany region [20].

This study confirmed the presence and diffusion of R. felis in cat and dog fleas (C. felis) from Italy, similar to other European countries [21]. The results also suggest that $R$. felis should be considered in the human differential diagnosis of any spotted-like fevers in Italy, especially if the patient is known to have been exposed to flea bites. Nonetheless, the role of mammals, mainly dogs and cats, in the epidemiology of this flea-borne infection needs further confirmatory evidence. To unravel the close relationship between $R$. felis and C. felis more studies are needed in rickettsial infection dynamics in the flea vector, which likely will further clarify the ecology and epidemiology of $R$. felis transmission in nature.

\section{Competing interests}

The authors declare that they have no competing interests in relation to this manuscript.

\section{Authors' contributions}

GC, FM and GM conceived and designed the experiments; $\mathrm{EP}$ and GM carried out the molecular genetic analyses; GM, CF, LR, GO and DO have made substantial contributions to acquisition of fleas and related data; GC and FM analysed and interpreted the data and wrote the paper; GO, LR and especially DO revised the article critically for important intellectual content.

\section{Acknowledgements}

Authors wish to thank Anna Granato and Silvia Ravagnan for their contribution to the molecular study, Giovanni Cattoli and Alessandra Drago for amplicons sequencing, Franco Mutinelli for suggestions in drafting the paper and Stefano Marangon (Istituto Zooprofilattico Sperimentale delle Venezie) for financial support. Authors are particular grateful to all the veterinarians who collaborated in collecting fleas and data. A special thank is due to Mauricio C. Horta and Marcelo B. Labruna who kicked off the study in Italy.

This article is published as part of Parasites \& Vectors Volume 2 Supplement I, 2009: Proceedings of the 4th International Canine Vector-Borne Disease Symposium. The full contents of the supplement are available online at http://www.parasitesandvectors.com/supplements/2/SI.

Publication of the supplement has been sponsored by Bayer Animal Health $\mathrm{GmbH}$.

\section{References}

I. Parola P, Davoust B, Raoult D: Tick- and flea-borne rickettsial emerging zoonoses. Vet Res 2005, 36(3):469-492.

2. Pérez-Osorio CE, Zavala-Velázquez JE, Arias León JJ, Zavala-Castro JE: Rickettsia felis as emergent global threat for humans. Emerg Infect Dis 2008, I4(7): 1019-1023.

3. Gillespie JJ, Ammerman NC, Beier-Sexton M, Sobral BS, Azad AF: Louse- and flea-borne rickettsioses: biological and genomic analyses. Vet Res 2009, 40:12.

4. Adams JR, Schmidtmann ET, Azad AF: Infection of colonized cat fleas, Ctenocephalides felis (Bouché), with a rickettsia-like microorganism. Am J Trop Med Hyg 1990, 43:400-409.

5. Bouyer DH, Stenos J, Valdes PC, Moron CG, Popov VL, ZavalaVelazquez JE, Foil LD, Stothard DR, Azad AF, Walker DH: Rickettsia felis: molecular characterization of a new member of the spotted fever group. Int J Syst Evol Microbiol 200I, 5 I:339-347.

6. Ogata H, Renesto P,Audic S, Robert C, Blanc G, Fournier PE, Parinello $H$, Claverie JM, Raoult D: The genome sequence of Rickettsia felis identifies the first putative conjugative plasmid in an obligate intracellular parasite. PloS Biology 2005, 3(8):e248.

7. Fournier P-E, Belghazi L, Robert C, Elkarkouri K, Richards AL, Greub G, Collyn F, Ogawa M, Portillo A, Oteo JA, Psaroulaki A, Bitam I, Raoult $D$ : Variations of plasmid content in Rickettsia felis. PLoS ONE 2008, 3(5):e2289.

8. Horta MC, Labruna MB, Durigon EL, Schumaker TT: Isolation of Rickettsia felis in the mosquito cell line C6/36. Appl Environ Microbiol. 2006, 72(2): I 705-I707.

9. Maioli G, Horta MC, Ogrzewalska M, Capelli G, Souza SO, Richtzenhain LJ and Labruna MB: First detection of Rickettsia felis in Ctenocephalides felis fleas in Italy. Clin Microbiol Infect (Suppl), in press.

10. Franc M: Puces et méthodes de lutte. Rev Sci Tech Off Int Epiz 1994, I3(4): 1019-1037.

I I. Labruna MB, Whitworth T, Horta MC, Bouyer DH, McBride JW, Pinter A, Popov V, Gennari SM, Walker DH: Rickettsia species infecting Amblyomma cooperi ticks from an endemic area for Brazilian spotted fever in the state of São Paulo, Brazil. J Clin Microbiol 2004, 42:90-98.

I2. Gilles J, Thomas F, Hellmann JK, Silaghi C, Pradel I, Friche Passos LM, Lengauer H, Pfister K: Rickettsia felis in fleas, Germany. Emerg Infect Dis 2008, I 4(8): I 294-I296.

13. Gilles J, Just FT, Silaghi C, Pradel I, Lengauer H, Hellmann K, Pfister K: Rickettsia felis in fleas, France. Emerg Infect Dis 2008, I 4:684-686.

14. Márquez FJ, Muniain MA, Rodríguez-Liebana JJ, Del Toro MD, Bernabeu-Wittel M, Pachón AJ: Incidence and distribution pattern of Rickettsia felis in peridomestic fleas from Andalusia, Southeast Spain. Ann NY Acad Sci 2006, I 078:344-346.

15. Azad AF, Sacci JB Jr, Nelson WM, Dasch GA, Schmidtmann ET, Carl M: Genetic characterization and transovarial transmission of a typhus-like Rickettsia found in cat fleas. Proc Natl Acad Sci USA 1992, 89:43-46.

16. Macaluso KR, Pornwiroon W, Popov VL, Foil LD: Identification of Rickettsia felis in the salivary glands of cat fleas. Vector Borne Zoonotic Dis 2008, 8(3):391-396.

17. Wedincamp J, Jr, Foil LD: Infection and seroconversion of cats exposed to cat fleas (Ctenocephalides felis, Bouché) infected with Rickettsia felis. J Vector Ecol 2000, 25: I 23-I 26.

18. Reif KE, Stout RW, Henry GC, Foil LD, Macaluso KR. Prevalence and infection load dynamics of Rickettsia felis in actively feeding cat fleas. PLoS ONE 2008, 3(7):e2805.

19. Ciceroni L, Pinto A, Ciarrocchi S, Ciervo A: Current knowledge of rickettsial diseases in Italy. Ann NY Acad Sci 2006, I078: I43-I 49.

20. Selmi M, Bertolotti L, Tomassone L, Mannelli A: Rickettsia slovaca in Dermacentor marginatus and tick-borne lymphadenopathy, Tuscany, Italy. Emerg Infect Dis 2008, I 4(5):817-820.

21. Brouqui P, Parola P, Fournier PE, Raoult D: Spotted fever rickettsioses in southern and eastern Europe. FEMS Immunol Med Microbiol 2007, 49:2-I2. 